\title{
Application and Comparison of Nonlinear Estimation Strategies on a Magnetorheological Hysteretic Force Model
}

\author{
Andrew Lee ${ }^{1}$ and S. Andrew Gadsden ${ }^{1}$ \\ ${ }^{1}$ College of Engineering and Physical Sciences \\ University of Guelph, Guelph, ON, Canada \\ Emails: alee32 and gadsden@uoguelph.ca
}

\begin{abstract}
The main goal of estimation theory is to minimize state error estimation while maintaining robustness. While the Kalman filter produces the optimal estimate for known linear systems with Gaussian noise, most electromechanical systems such as magnetorheological dampers contain nonlinearities. Nonlinear strategies such as the extended Kalman filter and smooth variable structure filter can be used to estimate the force produced by a damper given its velocity and acceleration and applied current. This paper compares the extended Kalman filter and the smooth variable structure filter on a magnetorheological damper that is subject to harmonic excitation. The purpose of this study is to apply and compare estimation methods that may be used in conjunction with a reliable controller for applications on a magnetorheological damper.
\end{abstract}

Keywords - Estimation strategies; extended Kalman flter; smooth variable structure filter; magnetorheological hysteresis

\section{INTRODUCTION}

Noise is inherently present in measurement data and is the product of sensor precision and environmental factors. State estimation is the process of determining the values of state variables from systems and measurements with uncertainty and noise. The purpose of estimation is to minimize the state estimation error while simultaneously being robust to disturbances, faults, and modeling uncertainty [1]. Reliable estimates of state parameters are necessary for safely controlling electro-mechanical systems such as a magnetorheological (MR) damper in real-time [2].

\section{A. Estimation Strategies}

Rudolf Kalman introduced a new approach to prediction problems and linear filtering in 1960. His work would eventually be referred to as the Kalman filter (KF) [2]. The KF uses sequential discrete-time measurements from a linear system model with Gaussian noise to produce an optimal state estimate [2]. The Kalman filter has numerous applications such as target tracking, signal processing and fault detection [2] [3] [4] [5] [6]. The Kalman gain minimizes the trace of the a posterior state error covariance matrix which is proven to produce the optimal state estimate for known linear systems with Gaussian noise [2] [3] [4].

The extended Kalman Filter (EKF) can be used to estimate the states of a nonlinear dynamic system. The filter uses local linearization of the system model at the operating point in order to calculate the corrective Kalman gain [2]. The EKF derivation is based on the Taylor series expansion of the nonlinear system model also known as the Jacobian [2]. However, if the system is highly nonlinear, the EKF solution may diverge from the true state trajectory.

The smooth variable structure filter (SVSF) is an extension of the KF that adds stability and robustness in the presence of disturbances, noise, and modeling uncertainties [3] [4]. The efficacy of the EKF and SVSF is compared when applied to a MR hysteretic force model.

\section{B. Magnetorheological Systems}

Magnetorheological (MR) fluids are a class of smart fluids that significantly change their viscosity when a magnetic field is applied. This intelligently controllable fluid allows for smooth force adjusting and repeatability [7]. Jacob Rabinov discovered the MR fluid effect in the 1940s and commercial MR products have recently attained low viscosities and high yield stresses [8]. MR fluids consist of micron-scale (1 to 10 $\mu \mathrm{m})$ magnetically polarizable particles suspended in a carrier medium such as mineral or silicon oil [9]. Surfactants are often used in the non-colloidal mixture in order to prevent settling of the suspended particles [10]. The magnetic particles typically make up between 20 and 60 percent of the MR fluid's volume [9].

When a magnetic field is applied to the MR fluid, the ferromagnetic particles rearrange to form linear structures and chains. The formation of these structures results in a change in the fluid's viscosity. The effect is almost instantaneous with a $100 \mathrm{kPa}$ yield stress that is achievable within a few milliseconds [11]. 
Increasing the strength of the magnetic field increases the yield strength of the MR fluid. This property has three main engineering application modes: flow (valve) mode, shear mode and squeeze mode [10]. In flow mode, the magnetic field is normal to the flow of the MR fluid and is typically used for linear dampers [10]. In shear mode, the magnetic field is normal to the displacement of the shear walls and is used for rotary dampers, breaks, and clutches [10]. Finally, squeeze mode utilizes a magnetic field that is parallel to the desired displacement of the containing walls. The squeeze mode provides large forces for relatively small displacements [10].

\section{Magnetorheological Dampers}

The quick response time of MR fluid behavior when exposed to a magnetic field is ideal for electromechanical devices such as actuators and dampers. Semi-active MR control devices have the versatility of active dampers while retaining the reliability of passive ones [10]. The two main engineering applications of MR fluids are linear and rotary dampers.

The main advantage of linear MR dampers is the controllability of the system damping by changing the input current. By changing the current, the magnetic induction in an orifice between two separated MR fluid chambers is regulated [10]. Linear MR dampers such as the one shown in Fig. 1 use the MR fluid in flow mode to treat the orifice as a valve for the MR fluid. One of the first applications of linear MR dampers in the automotive industry is its use as a secondary suspension element for on and off highway vehicles [8]. By controlling the damping arrangement, the excitation frequency and vibration transmission can be adjusted as desired [8].

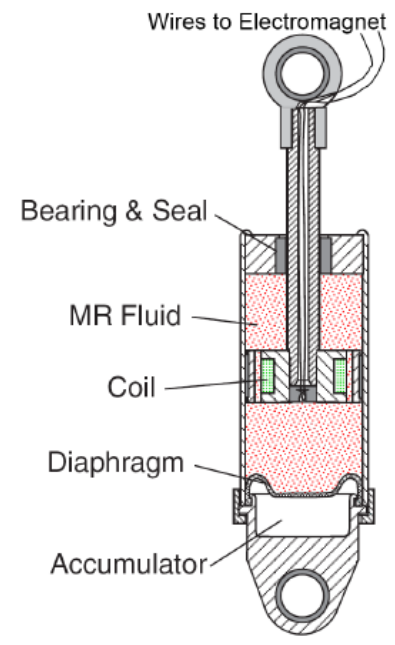

Figure 1. Diagram of single ended linear MR damper [10]

\section{Smooth Variable Structure Filter}

The SVSF uses an upper bound on the level of unmodeled dynamics and noise in order to achieve robustness to disturbances and modeling uncertainties. Like the KF, the SVSF is uses a system model and can be applied to both differentiable linear or nonlinear dynamic equations $[2,12]$.
The SVSF estimation process is similar to the KF. However, it presents a novel method of gain calculation [2, 13]. The predicted state estimates $\hat{x}_{k+1 \mid k}$ and state error covariance $P_{k+1 \mid k}$ are first calculated as per the following:

$$
\begin{gathered}
\hat{x}_{k+1 \mid k}=A \hat{x}_{k \mid k}+B u_{k} \\
P_{k+1 \mid k}=A P_{k \mid k} A^{T}+Q_{k} .
\end{gathered}
$$

Utilizing the predicted state estimates $\hat{x}_{k+1 \mid k}$, the corresponding predicted measurements $\hat{z}_{k+1 \mid k}$ and measurement errors $e_{z, k+1 \mid k}$ may be calculated:

$$
\begin{gathered}
\hat{z}_{k+1 \mid k}=C \hat{x}_{k+1 \mid k} \\
e_{z, k+1 \mid k}=z_{k+1}-\hat{z}_{k+1 \mid k} .
\end{gathered}
$$

The SVSF gain is a function of the following: 1) a priori and the a posteriori measurement errors $e_{z, k+1 \mid k}$ and $e_{z, k \mid k} ; 2$ ) the smoothing boundary layer widths $\psi ; 3$ ) and the 'SVSF' memory or convergence rate $\gamma$. The SVSF gain $K_{k+1}$ is defined as follows [2] [3]:

$$
\begin{gathered}
K_{k+1}=C_{k}^{+} \operatorname{diag}\left[\left(\left|e_{z_{k+1 \mid k}}\right|+\gamma\left|e_{z_{k \mid k}}\right|\right) \circ\right. \\
\left.\operatorname{sat}\left(\bar{\psi}^{-1} e_{z_{k+1 \mid k}}\right)\right] \operatorname{diag}\left(e_{z_{k+1 \mid k}}\right)^{-1}
\end{gathered}
$$

where $\circ$ signifies Schur (or element-by-element) multiplication and the superscript + refers to the pseudoinverse of a matrix [2] [3]. The saturation function of (5) is defined by the following:

$$
\begin{aligned}
& \operatorname{sat}\left(\bar{\psi}^{-1} e_{z_{k+1 \mid k}}\right) \\
& =\left\{\begin{aligned}
1, & e_{z_{i}, k+1 \mid k} / \psi_{i} \geq 1 \\
e_{z_{i}, k+1 \mid k} / \psi_{i}, & -1<e_{z_{i}, k+1 \mid k} / \psi_{i}<1 \\
-1, & e_{z_{i}, k+1 \mid k} / \psi_{i} \leq-1 .
\end{aligned}\right.
\end{aligned}
$$

where $\bar{\psi}^{-1}$ is a diagonal matrix constructed from the elements of the smoothing boundary layer vector $\psi$ :

$$
\bar{\psi}^{-1}=\left[\begin{array}{ccc}
\frac{1}{\psi_{1}} & 0 & 0 \\
0 & \ddots & 0 \\
0 & 0 & \frac{1}{\psi_{m}}
\end{array}\right] .
$$

The state estimates $\hat{x}_{k+1 \mid k}$ and state error covariance matrix $P_{k+1 \mid k}$ are updated respectively as per the following:

$$
\begin{gathered}
\hat{x}_{k+1 \mid k+1}=\hat{x}_{k+1 \mid k}+K_{k+1}\left[z_{k+1}-C \hat{x}_{k+1 \mid k}\right] \\
P_{k+1 \mid k+1}=\left[I-K_{k+1} C\right] P_{k+1 \mid k}\left[I-K_{k+1} C\right]^{T} \\
+K_{k+1} R_{k+1} K_{k+1}^{T} .
\end{gathered}
$$

Finally, the updated measurement estimate $\hat{z}_{k+1 \mid k+1}$ and measurement errors $e_{z, k+1 \mid k+1}$ are calculated, and are used in later iterations:

$$
\begin{gathered}
\hat{z}_{k+1 \mid k+1}=C \hat{x}_{k+1 \mid k+1} \\
e_{z, k+1 \mid k+1}=z_{k+1}-\hat{z}_{k+1 \mid k+1} .
\end{gathered}
$$


The existence subspace denoted by the dotted black line shown in Figure 2. represents the level of uncertainty present in the estimation process, in terms of modeling errors or the presence of noise [3]. The width of the existence space is a function of the uncertain dynamics associated with the inaccuracy of the internal model of the filter as well as the measurement model, and varies with time [2] [3] [4].

While this value is not precisely known, an upper bound may be selected based on a priori knowledge [3]. The estimated state trajectory is smoothed when the smoothing boundary layer is defined larger than the existence subspace boundary [3]. If the smoothing term is too small, however, chattering oscillations persist due to the uncertainties being underestimated [3].

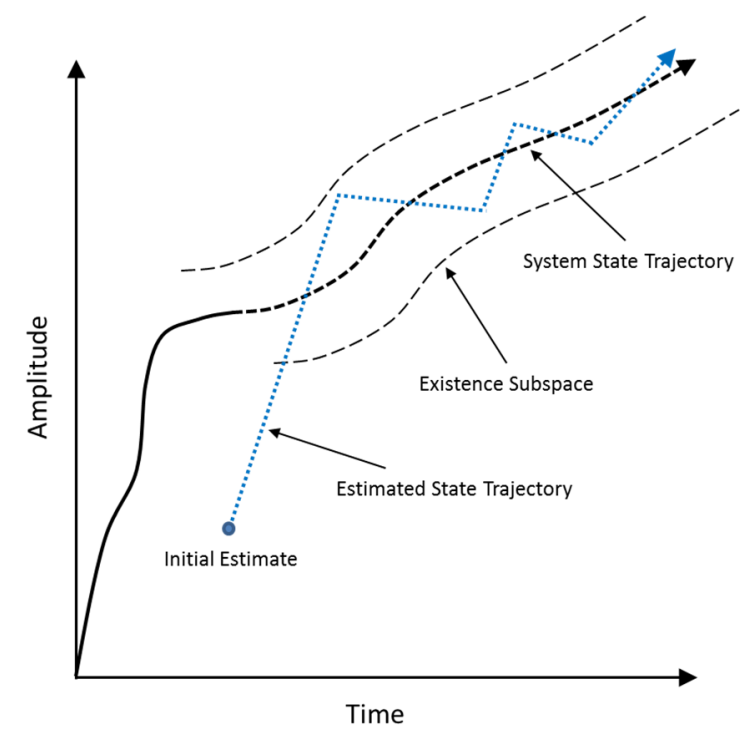

Figure 2. SVSF concept with existence subspace boundary layer [5]

\section{MagnetorheOlogical Hysteresis ModeL}

The force-velocity relationship of an MR damper is strongly dependent upon the current. However, variations in current do not appear to affect the width of the hysteresis loop [14]. The damping force of an MR damper can be described by the following:

$$
f_{d}=f_{i}(i) f_{h}(x, \dot{x}, \ddot{x})
$$

where $f_{i}$ is a function of current, $i$, and $f_{h}$ describes the hysteretic behavior of the piston displacement, velocity, and acceleration denoted by $x, \dot{x}$, and $\ddot{x}$ respectively [14].

The nonlinear incremental current is characterized by an asymmetric sigmoid function with a bias in the horizontal axis given by:

$$
f_{i}(i)=1+\frac{k_{2}}{1+e^{-a_{2\left(i+I_{0}\right)}}}-\frac{k_{2}}{1+e^{-a_{2} I_{0}}} ; i \geq 0
$$

where $k_{2}, a_{2}$, and $I_{0}$ are constants obtained through measured data.
A model composed of two polynomial functions corresponding to positive and negative piston acceleration was used to test the efficacy of the EKF and SVSF. This model was first proposed by Choi et al [15]. The polynomial hysteresis model can by:

$$
f_{h}=\left(\sum_{k=0}^{n} b_{k} \dot{x}(t)^{k}\right) ; n=6
$$

where $b_{k}$ represents the polynomial coefficient constants obtained through experimentation, $k$ is polynomial exponent, and $n$ is the order of the polynomial. Combing (12) and (13) the piece-wise hysteric damping force function becomes [14]:

$$
f_{d}=\left\{\begin{array}{cc}
f_{i}(i)\left(\sum_{k=0}^{6} b_{u k} \dot{x}(\dot{t})^{k}\right) ; & \ddot{x}<0 \\
f_{i}(i)\left(\sum_{k=0}^{6} b_{d k} \dot{x}(t)^{k}\right) ; & \ddot{x}>0 \\
f_{i}(i)\left(\sum_{k=0}^{6} \frac{1}{2}\left(b_{u k}+b_{d k}\right) \dot{x}(t)^{k}\right) ; & \ddot{x}=0
\end{array}\right.
$$

where $b_{u k}$ and $b_{d k}$ represent the coefficients of the upper and lower polynomial respectively. In order to ensure the convergence of the two polynomial functions near the extremities, when the piston acceleration is 0 , the damping force is calculated as the average of the upper and lower polynomial functions [14]. The values for the model parameters are shown in Table I.

TABLE I. PARAMETERS FOR HYSTERESIS MODEL

\begin{tabular}{|c|l|}
\hline Parameter & \multicolumn{1}{|c|}{ Values } \\
\hline$k_{2}$ & 3.10 \\
$a_{2}$ & 18.53 \\
$I_{0}$ & -0.15 \\
$b_{u 0}$ & 0.06 \\
$b_{u 1}$ & 4.97 \\
$b_{u 2}$ & -71.29 \\
$b_{u 3}$ & -1461.82 \\
$b_{u 4}$ & 23232.45 \\
$b_{u 5}$ & 190972.68 \\
$b_{u 6}$ & -2319223.80 \\
$b_{d 0}$ & -0.07 \\
$b_{d 1}$ & 5.03 \\
$b_{d 2}$ & 78.00 \\
$b_{d 3}$ & -1549.09 \\
$b_{d 4}$ & -27398.44 \\
$b_{d 5}$ & 210738.82 \\
$b_{d 6}$ & 3017864.40 \\
\hline
\end{tabular}




\section{EKF AND SVSF ASSESSMENT}

The EKF and SVSF were used to estimate the position, velocity, acceleration, and damping force of a linear MR damper similar to the one shown in Fig 1. The MR damper is subjected to harmonic excitation at a frequency of $1.5 \mathrm{~Hz}$ and an amplitude of $6.25 \mathrm{~mm}$. The input current of the MR damper is $1.0 \mathrm{~A}$. The system $(\mathrm{Q})$ and measurement $(\mathrm{R})$ noise covariance matrices are given by the following:

$$
\begin{aligned}
& Q=\operatorname{diag}[1 \mathrm{e}-8 \mathrm{1e}-6 \text { 1e }-41 \mathrm{e}-3] \\
& R=\operatorname{diag}[1 \mathrm{e}-7 \text { 1e-5 } 1 \mathrm{e}-32 \mathrm{e}-2] \text {. }
\end{aligned}
$$

The simulation was run for a duration of 10 cycles totaling approximately 6.67 seconds. The velocity and damping force over this duration is depicted in Fig. 3.
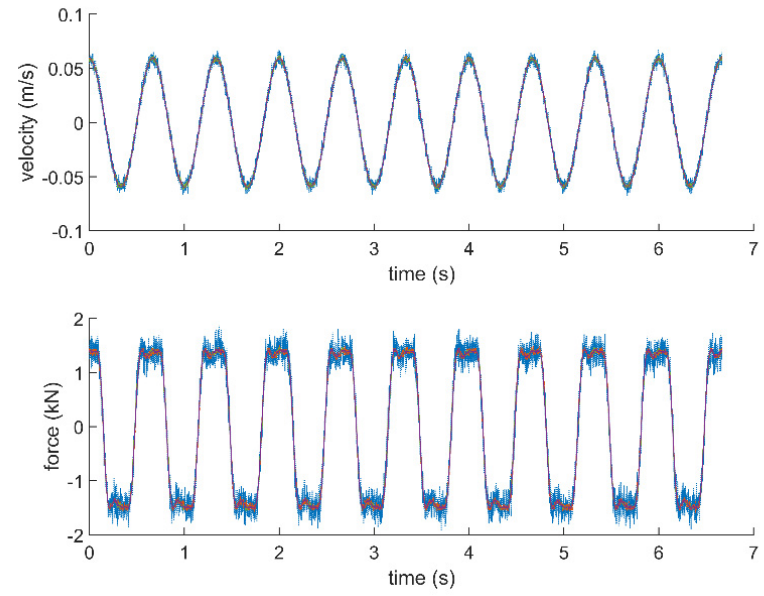

Figure 3. Velocity and Damping Force of MR Damper

The boundary layer for the SVSF was optimized using a grid search across all states while trying to minimize the normalized error squared. The boundary layer vector contains the following values:

$$
\psi=\operatorname{diag}\left[\begin{array}{llll}
0.02 & 0.14 & 4.0 & 4.0
\end{array}\right] .
$$

A close-up of the velocity and force estimates are shown in Fig 4. and Fig 5. respectively. These figures are able to show a representation of the efficacy of each filter by visually comparing the filtered estimates with the true states. The SVSF is significantly more robust to measurement noise and is able to produce smoother state estimates when compared to the EKF as shown in Fig. 4.

The hysteretic force-velocity curve is shown in Fig. 6. The SVSF produces a significantly smoother characterization than the sensor measurement and outperforms the EKF as shown by the normalized error squared plot in Fig 7.

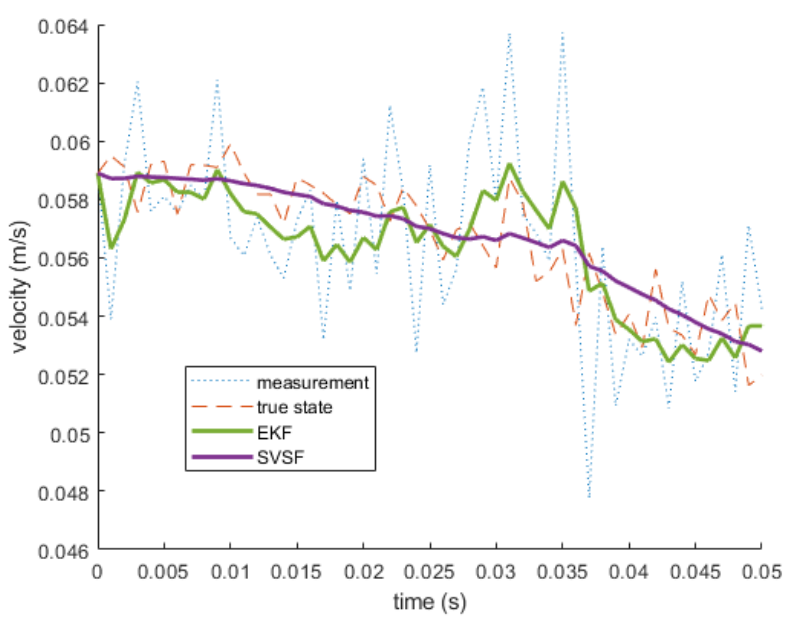

Figure 4. Velocity of MR Damper (close-up)

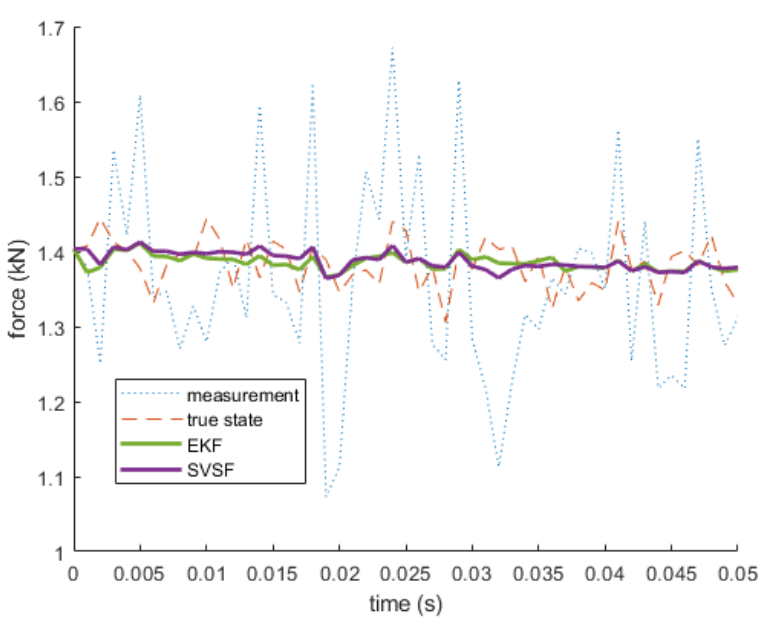

Figure 5. Force of MR Damper (close-up)

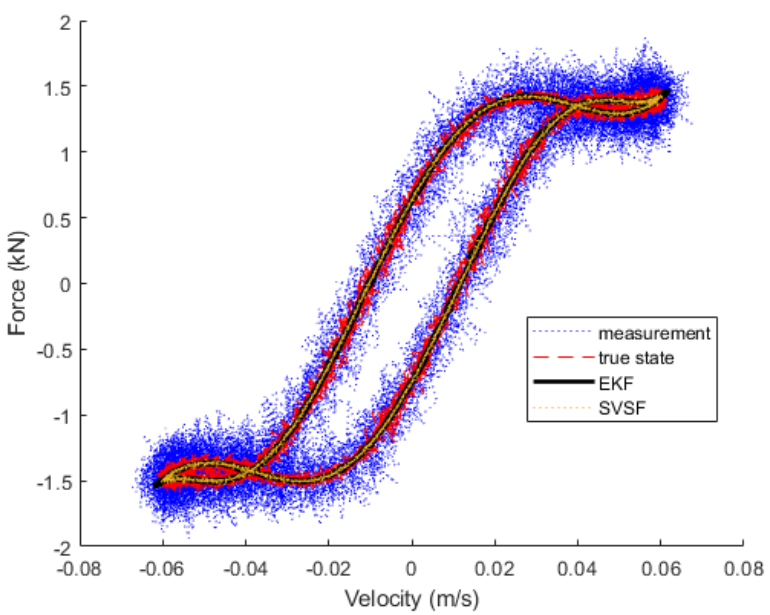

Figure 6. Hystereitc Force-Velocity Curve 


\section{REFERENCES}

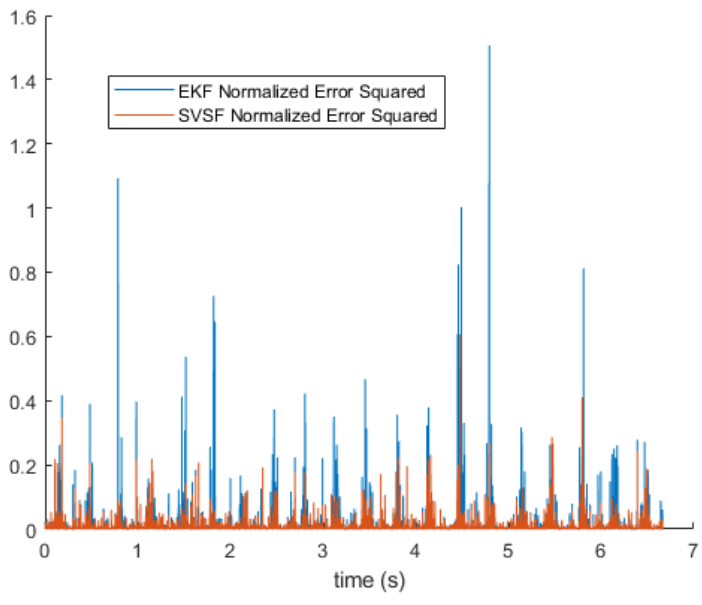

Figure 7. Normalized Error Squared

The root mean squared error (RMSE) of the estimate with respect to the true state value was calculated to precisely compare the efficacy of the EKF and SVS. Table II shows the RMSE values for both filters.

TABLE II. ROOT MEAN SQUAREd ERROR

\begin{tabular}{|l|c|c|c|c|}
\hline \multirow{2}{*}{ Filter } & \multicolumn{4}{|c|}{ State } \\
\cline { 2 - 5 } & $\begin{array}{c}\text { Position } \\
(\boldsymbol{m})\end{array}$ & $\begin{array}{c}\text { Velocity } \\
(\boldsymbol{m} / \mathbf{s})\end{array}$ & $\begin{array}{c}\text { Acceleration } \\
\left(\boldsymbol{m} / \mathbf{s}^{2}\right)\end{array}$ & $\begin{array}{c}\text { Force } \\
(\boldsymbol{P a})\end{array}$ \\
\hline EKF & $1.483 \mathrm{e}-4$ & $1.412 \mathrm{e}-3$ & $9.596 \mathrm{e}-3$ & $4.641 \mathrm{e}-2$ \\
\hline SVSF & $1.075 \mathrm{e}-4$ & $1.088 \mathrm{e}-3$ & $10.538 \mathrm{e}-3$ & $4.375 \mathrm{e}-2$ \\
\hline
\end{tabular}

Based on the RMSE values, the SVSF outperformed the EKF in terms of position, velocity, and damping force estimates. While the SVSF did not outperform the EKF, in acceleration estimation, the velocity and force estimates are the most crucial states when implementing a controller for the MR damper. Thus, the SVSF is the preferred model-based filter in this scenario. However, the EKF may be considered easier to implement as it does not require tuning of boundary layer parameters (although it does still require the use of a linearization matrix to approximate the nonlinearities).

\section{CONCLUSIONS}

In this paper, a hysteresis force model of a linear magnetorheological damper was studied using the EKF and SVSF. In this study, the SVSF was able to significantly mitigate system and measurement noise and produced smaller state estimation errors compared with the EKF. The SVSF can be used to obtain reliable estimates of state parameters which are necessary for controlling electro-mechanical systems. Future studies will use the SVSF for fault detection on the MR damper due to wear on the diaphragm or inconsistencies in the current supply.
[1] H. Afshari, S. A. Gadsden and S. R. Habibi, "Gaussian Filters for Parameter and State Estimation: A Review of Theory and REcent Trends," Sginal Processing, vol. 135, no. C, pp. 218-238, 2017.

[2] A. S. Lee, S. A. Gadsden and S. A. Wilkerson, "An adaptive smooth variable structure filter based on the static multiple model strategy," SPIE Signal Processing, Sensor/Information Fusion, and Target Recognition, no. XXVIII, 2019.

[3] B. Ristic, S. Arulampalam and N. Gordon, Beyond the Kalman Filter: Particle Filters for Tracking Applications, Boston: Artech House, 2004.

[4] S. Haykin, Kalman Filtering and Neural Networks, New York: John Wiley and Sons, Inc., 2001.

[5] S. A. Gadsden, "Smooth Variable Structure Filtering: Theory and Applications," McMaster University, Hamilton, 2011.

[6] S. A. Gadsden, M. Al-Shabi and S. R. Habibi, "Estimation Strategies for the Condition Monitoring of a Battery System in a Hybrid Electric Vehicle," ISRN Signal Processing, 2011.

[7] D. Guth and J. Mas, "Energy-Efficient Clutch with Optimized Torque Density," Smart Materials, Adaptive Structures and Intelligent Systemd, no. SMASIS2013, 2013.

[8] A. Olabi and A. Grunwald, "Design and Application of MagnetoRheological Fluid," Materials and Design, vol. 28, no. C, pp. 26582664, C.

[9] A. Milecki, "Investigation and Control of Magneto-Rheological Fluid Dampers," International Journal of Machine Tools and Manufacture, vol. 41, no. C, pp. 379-391, 2001.

[10] A. Spaggiari, "Properties and Applications of Magnetorheological Fluids," Frattura ed Integrità Strutturale, vol. 23, no. 10.3221/IGFESIS.23.06, pp. 57-61, 2013.

[11] P. Yadmellat and M. R. Kermani, "Adaptive Hysteresis Compensation for a Magneto-Rheological Robot," IEEE Intelligent Robots and Systems IROS, no. DOI: 978-1-4673-6358-7/13, 2013.

[12] S. A. Gadsden and A. S. Lee, "Advances of the Smooth Variable Structure Filter: Square-Root and Two-Pass Formulations," Journal of Applied Remote Sensing, vol. 11, no. 1, 2017.

[13] S. A. Gadsden and S. R. Habibi, "Model-Based Fault Detection of a Battery System in a Hybrid Electric Vehicle," in IEEE Vehicle Power and Propulsion Conference (VPPC), Chicago, Illinois, 2011.

[14] X. Q. Ma, S. Rakesha and C. Su, "Development and Relative Assessments of Models for Characterizing the Current Dependent Hysteresis Properties of Magnetorheological Fluid Dampers," Journal of Intelligent Material Systems and Structures, vol. 18, no. DOI: 10.1177/1045389X06067118, pp. 487-502, 2007.

[15] S. B. Choi, B. K. Lee and Y. P. Park, "A Hysteresis Model for the Field-dependent Damping Force of a Magnetorheological Damper," Journal of Sound and Vibration, vol. 2, no. 245, p. 375, 2001

[16] W. Youn and S. A. Gadsden, "Combined Quaternion-Based Error State Kalman Filtering and Smooth Variable Structure Filtering for Robust Attitude Estimation," IEEE Access, vol. 7, pp. 148989-149004, 2019.

[17] S. A. Gadsden, S. R. Habibi and T. Kirubarajan, "Kalman and Smooth Variable Structure Filters for Robust Estimation," IEEE Transactions on Aerospace and Electronic Systems, vol. 50, no. 2, pp. 1038-1050, 2014.

[18] J. Goodman, S. A. Wilkerson, C. Eggleton and S. A. Gadsden, "A multiple model adaptive SVSF-KF estimation strategy," in 2019 SPIE Signal Processing, Sensor/Information Fusion, and Target Recognition XXVIII, Baltimore, Maryland, 2019.

[19] C. Bourque, A. S. Lee, E. Hill, A. Bardelcik, S. A. Gadsden and M. Biglarbegian, "Kalman Filtering and PID Control of an Inverted Pendulum Robot," in CSME International Congress, London, Ontario, 2019. 